\title{
Organizaciones con auto-gobierno y marco legal: ¿jinergia?
}

\author{
Jacinta Palerm-Viqueira \\ Colegio de Postgraduados \\ jacinta.palerm@gmail.com
}

\begin{abstract}
Resumen. En este trabajo se explora la relación entre la auto-gestión o auto-gobierno de sistemas de riego y el reconocimiento gubernamental sobre derechos de los regantes a auto-organizarse. En el caso de México los cambios frecuentes en la legislación sobre el marco jurídico para las organizaciones de regantes harían pensar en su mayor fragilidad. Sin embargo lo que encontramos en unos 20 estudios de caso es que las organizaciones funcionan en la informalidad, lo que implica que en todo caso se hacen invisibles a los ojos del Estado.
\end{abstract}

Palabras clave: Reconocimiento mínimo al derecho a organizarse; autogobierno; informalidad; legislación.

\begin{abstract}
The relationship between irrigation system self-governance or self-management and government recognition of irrigator's rights to self-organize is explored. In XXth century Mexico frequent innovations on the legislation dealing with the legal framework for water user's organizations should impact the organizations negatively. However, the evidence from some 20 case studies is that the water users' organizations are capable of working informally (without a government approved official organization). This has the implication that, for the government, the on-the-ground water users' organizations are invisible.
\end{abstract}

Keywords: Minimal recognition of rights to organize; self-governance; informality; legislation.

\section{Introducción}

Ya no hay dudas sobre la capacidad de los regantes de auto-gobernarse (Maass \& Anderson, 1976; Glick, 1970; Hunt, 1997) aunque los grandes sistemas de riego parecen requerir personal profesionalizado y por tanto una gestión burocrática, mientras que sistemas más pequeños pueden ser operados por los propios regantes (Worster, 1985; Vaidyanathan, 2009).

Sin embargo, la relación entre el tamaño del sistema de riego y el tipo de gestión (burocrática o no burocrática) en los sistemas de riego auto-gobernados está abierta a discusión. Erickson argumenta que la gente común es capaz de construir y manejar estructuras complejas sin burocracias ni jerarquías (Erickson, 2006).

No obstante, parece haber un punto de inflexión entre la gestión burocrática y no burocrática. Una revisión sobre los estudios de caso, incluyendo casos de regiones tan diversas como México, España, los EE.UU., Nepal y Japón, sitúa empíricamente el punto de inflexión en alrededor de 15.000 a 20.000 hectáreas (37.065 a 49.421 acres) regadas por el mismo sistema; es decir, los sistemas de riego más grandes pueden ser auto-gobernados pero dependen de una gestión burocrática (Palerm-Viqueira, 2000, 2006).

A pesar de la capacidad de los regantes para auto-gobernarse, hay importantes diferencias regionales alrededor del mundo entre los sistemas de riego auto-gobernados. Nirmal Sengupta, un erudito indio, sugiere que las diferencias regionales del mundo en el desarrollo de 
sistemas de riego auto-gestionados están relacionadas con marcos jurídicos estables de larga duración a favor de la autogestión (Sengupta, 2002). La propuesta de Sengupta está en consonancia con uno de los principios de diseño de Ostrom, el "reconocimiento mínimo de los derechos a organizarse". El argumento básico de Ostrom parece ser el impacto (negativo) de la interferencia del gobierno al socavar o contradecir las reglas locales, y no una falta de capacidad de autogestión per se (Ostrom, 1993, 2009, 2011).

Hace un par de años, y centrándonos en los argumentos de Sengupta, realizamos un seguimiento histórico sobre la correlación entre la presencia de sistemas de riego autogobernados y la estabilidad en el largo plazo de marcos jurídicos favorables al autogobierno. Con base en los casos de Perú, Chile, la provincia de Mendoza (Argentina) y México, así como en el caso de España, concluimos que hay una fuerte correlación, tal y como Sengupta encontró en su comparación de la India, Japón y Filipinas (PalermViqueira, 2010).

Posteriormente a la realización de esta comparación histórica a nivel país, decidimos echar un vistazo más de cerca al caso de México siguiendo una estrategia de estudios de caso con cierta profundidad histórica donde pudiéramos determinar los efectos sobre el autogobierno de los cambios en el marco legal. En México, el marco legal para el auto-gobierno de sistemas de riego sufrió cambios a lo largo del siglo XX, hay nuevas leyes emitidas en 1910, 1926, 1929, 1934, 1972 y 1992 (Palerm-Viqueira, 2005). En el mismo período de tiempo, también cambiaron políticas públicas en materia de gestión pública directa de los sistemas de riego, favoreciendo la expansión o contracción (Palerm-Viqueira, 2009).

En conjunto tenemos información de unos 20 estudios de caso que abarcan alrededor de 85.000 hectáreas (210.039 acres) de superficie de riego. Cabe notar que algunos de los estudios de caso comprenden más de un sistema en el mismo tramo de río. El rango de tamaños de los sistemas de riego va de unas 9.000 hectáreas $(22.239$ acres) (con cuatro casos), a sistemas pequeñitos que riegan unos pocos cientos de hectáreas.

Interesa señalar que, al inicio del proyecto, esperábamos encontrar fragilidad ante la pérdida del marco legal, además la expectativa era que los sistemas de riego más grandes y con mayor número de usuarios -en comparación con sistemas pequeños y con pocos usuarios- iban a presentar una situación de mayor fragilidad.

Sin embargo, lo que encontramos fue, en primer lugar, un número considerable de organizaciones no oficiales y, por no oficiales, queremos decir que no cumplen con el marco legal vigente; en segundo lugar, una impresionante resiliencia de las organizaciones. Los estudios de casos muestran que la organización informal es bastante extensa y exitosa. Los regantes han logrado no sólo mantener sus organizaciones, sino también añadir un segundo o tercer nivel organizativo. La visibilidad de las organizaciones, más que su existencia parece ser el problema. 


\subsection{Una breve nota metodológica.}

1.1.1 La unidad de observación: el sistema de riego y SU organización (y no la organización y su o sus sistemas de riego). El paso más difícil en todos los estudios de caso fue, probablemente, el dejar al lado la versión "oficial" de la organización de riego, y mirar a la organización existente in situ para un sistema de riego físico dado. Esta dificultad se agrava cuando, con demasiada frecuencia, la organización formal y el sistema de riego no son isomorfos.

Desde el punto de vista de la organización formal (oficial), el estudio de caso pudiera abarcar sistemas que no comparten la misma infraestructura, o incluso que no comparten la misma agua del río, o que cubren sólo una porción del sistema de riego (Palerm-Viqueira, 2001; Hunt et al., 2005; Hunt, 2007).

La decisión para todos los estudios de caso fue mirar a la organización in situ para un dado sistema de riego. Un ejercicio extremadamente útil es desprenderse de la terminología nacional: distrito de riego, junta de aguas, urderal, unidad de riego ...

1.1.2 La continuidad organizativa. Para poder hablar de persistencia de una organización es evidente que hay que rastrear a la organización en el tiempo. La persistencia de la infraestructura o del regadío no necesariamente denota la persistencia de la organización. En México hay varias rupturas organizativas, como son la desamortización y el reparto agrario, pero también los cambiantes marcos legales y lo que parece ser una política de estado para ignorar la historia que da como primera impresión que todos los sistemas tienen organizaciones nuevecitas, datadas a partir de 1992 -incluso con títulos de concesión nuevecitos.

\section{Los estudios de caso}

Para fines de presentación, vamos a agrupar los casos por periodo de origen de una organización que persiste hasta la fecha del estudio de caso. De esta manera visibilizamos la antigüedad de la organización y contrastamos con la legislación vigente, cuando menos durante el siglo XX, sobre organizaciones de regantes.

\subsection{La persistencia de las organizaciones muy antiguas, que datan de la época colonial}

Casos de este tipo no son frecuentes. Aunque los sistemas de riego físicos se remontan a tiempos prehispánicos y coloniales, hay una falta de continuidad de la organización social debido a los cambios en la tenencia de la tierra: primero la concentración de propiedad a finales del siglo XIX que desplazó las tenencias comunitarias campesinas, seguido por la reforma agraria en los años de 1930 que dispersó propiedad. Sin embargo, en tres de los estudios de caso hubo poca interrupción relacionada con cambios en la tenencia de la tierra y, por lo tanto, hubo persistencia de organizaciones antiguas. No obstante, las organizaciones de regantes han tenido que lidiar con cambios impuestos por el gobierno, tales como:

a) integración a un Distrito de Riego gestionado por el gobierno, desplazando la auto-gestión

b) nuevos límites: la integración de varios sistemas en una organización de todo el río. 
Juárez. La antigua sección del Distrito de Riego del valle de Juárez data de la época colonial. En el siglo XIX, la gestión parece haber estado formalmente en las manos del municipio. En el periodo de la reforma agraria, la tenencia de la tierra no cambió; sin embargo, el regadío contrajo unas 7,000 a 1,800 hectáreas entre 1896 y el 2007. A principios del siglo $\mathrm{XX}$, los regantes fueron investidos formalmente con la administración del sistema. En 1934, el sistema de riego se incorporó a la lista de Distritos de Riego, pero, en breve, en 1939, la gestión formalmente se enviste en la organización de regantes. En algún momento, tal vez en la década de los 70's por los cambios en la legislación que señalan que la administración de los Distritos de Riego es asunto del gobierno federal, las autoridades del Distrito trataron de tomar control de la acequia madre, pero los regantes negaron acceso a los funcionarios. Esta situación continuó hasta finales de los 90's, con la transferencia del Distrito de Riego. Unos 20 años de organización de regantes informal. Incluso con el proceso de transferencia, los regantes estaban negociando las condiciones, al sentir que el sistema le pertenecía a ellos, y no al gobierno (Arteaga, 1931; Rodríguez-Haros \& Palerm-Viqueira, 2007; Palerm-Viqueira, 2009).

Valle de Santiago. El sistema de riego de Valle de Santiago data de la época colonial; la datación es precisa puesto que el sistema depende del lago artificial Yuriria. El sistema riega unas 10.000 hectáreas. Los reglamentos sobre este sistema datan de 1611, 1780, 1850, 1916 y 1930. La gobernanza del sistema de riego es aparentemente excepcional para México, por la característica de un gobierno unificado. Es típico en México colonial y del XIX organizaciones horizontales (Palerm-Viqueira, 2010; Sandré \& Sánchez, 2011). Con la reforma agraria hubo poca alteración en la tenencia de la tierra y un núcleo de regantes antiguos conservó sus tierras, pero el sistema fue integrado en el Distrito de Riego Alto Lerma en 1934, y en 1938 la auto-gestión fue oficialmente suprimida en el Distrito. La resistencia a la supresión de las organizaciones de auto-gobierno es desconocida, sin embargo, los conflictos entre las autoridades del Distrito de Riego y varios organizaciones de regantes están documentados (Santos, 2006; Palerm-Viqueira, 2009). Los viejos regantes del Valle de Santiago aparentemente mantuvieron solidaridad y tienen una historia de negociaciones exitosas con las autoridades del distrito -algunos informantes locales dicen que las autoridades del Distrito les tenían miedo. Con la transferencia del Distrito en la década de 1990, el módulo Valle de Santiago es un caso modelo de éxito de la transferencia. La administración, sin embargo, es de tipo burocrático. La supresión del auto-gobierno de 1938 hasta la década de 1990: es decir, unos 50 años (Palerm-Viqueira, Sandré, Rodríguez-Haros, \& Caletteet, 2004; Palerm-Viqueira, 2009; Garma, 2006; Sandré \& Sánchez, 2011; Palerm-Viqueira \& Martínez-Saldaña, 2013)

Cuautitlán. El sistema de riego de Cuautitlán es prehispánico, datado desde la época en que el curso del río Cuautitlán fue modificado artificialmente. Los regantes pertenecen a antiguas comunidades indígenas y la reforma agraria sólo reforzó dichas comunidades. El sistema de riego de Cuautitlán, en el siglo XIX, tenía una administración formal municipal, o al menos la parte del sistema abarcada por el municipio. En la década de 1920, el gobierno propuso una administración de auto-gobierno pero unificando todos los sistemas que tomaban agua del río; los regantes de los distintos sistemas rehusaron la centralización, incluso cuando el gobierno propuesto era local. Uno a uno, los sistemas se las arreglaron para separarse de la organización centralizada y conseguir que su propia organización fuera reconocida oficialmente. Debido a los marcos legales, estas organizaciones han cambiado nombres, pero han mantenido su misma organización, salvo tal vez a nivel comunitario, 
donde la administración por el ejido fue reemplazada por un comité de riego ad hoc. En la década de los 70's, los tres sistemas que tomaban agua de la misma presa (Cuautitlán, con unas 7.000 hectáreas y otros dos pequeños sistemas, dando un total de aproximadamente 9.000 hectáreas (22.239 acres)) establecieron un comité no oficial. Este comité ha durado desde la década de los 70's hasta la fecha, es decir unos 40 años. El personal para la operación se nombra por elección entre los mismos regantes en el nivel de comité de los tres sistemas, de cada sistema y de cada comunidad. Los sistemas, sin embargo, probablemente no durarán mucho más debido a la expansión urbana (Sandré, 2012; Palerm-Viqueira \& Martínez-Saldaña, 2013).

\subsection{La persistencia de las organizaciones a principios del siglo $\mathrm{XX}$, creadas después de la reforma agraria, pero que fueron formalmente suprimidas, o cuyo marco legal no era vigente.}

Los nuevos regantes, beneficiarios de la reforma agraria, crearon, con el apoyo del gobierno, nuevas organizaciones dentro del marco legal vigente. Sin embargo, debido a la incorporación de los sistemas a distritos de riego y a la expansión de la administración gubernamental o debido a cambios en el marco legal, estas organizaciones perdieron formalidad. En este grupo de casos, hemos encontrado poco a nada de continuidad con organizaciones anteriores del siglo XIX. La reforma agraria sustituyó a las grandes haciendas con una multitud de regantes. Sin embargo, sospechamos que puede haber una continuidad en el nivel de comunidad, cuando menos la memoria histórica de derechos del agua.

Ixmiquilpan. El Distrito de Riego de Ixmiquilpan data de 1942, y está vinculado a nuevas obras, aunque varias obras de riego en el área son mencionadas en el siglo XVIII. El Distrito comprende tres sistemas de riego que riegan unas 3,500 hectáreas. La administración del Distrito se entregó a los regantes. Sin embargo, en la década de los 70's, debido a cambios en el marco legal, funcionarios del gobierno llegaron a tomar el control del Distrito de Riego. Los regantes se negaron y les aventaron piedras a los ingenieros -este evento nos fue narrado tanto por uno de los ingenieros apedreados, como por los regantes. Desde la década de 1970, los regantes han mantenido el control sobre el sistema de irrigación. En los 90's, con las políticas de trasferencia, los regantes de Ixmiquilpan se vieron obligados a exigir su status como Distrito de Riego, ya que éste estaba vinculado a inversiones del gobierno para modernizar la infraestructura hidráulica. Extrañamente ya no estaban en la lista de Distritos. El auto-gobierno, al margen del marco legal duró desde 1970 hasta la década de 1990, unos 30 años. (Rodríguez-Haros \& Palerm-Viqueira, 2007; Palerm-Viqueira, 2009).

Nazas. Los sistemas de riego de La Laguna fueron integrados en un Distrito de Riego en la década de 1930. Desde finales del siglo XIX, autoridades gubernamentales manejaron el río Nazas, y posteriormente las presas de almacenamiento; sin embargo cada sistema de riego era auto-gobernado. Con la reforma agraria se mantuvo el mismo esquema y en cada uno de los sistemas se puso en marcha una organización de regantes. Sin embargo, en 1962, la auto-gestión se suprimió en todo el Distrito de Riego y en la parte baja del Distrito se construyó una nueva red de canales. En la parte alta del río Nazas (y del Distrito), los pequeños sistemas no se modificaron. En total estos pequeños sistemas, que van desde unos pocos cientos de hectáreas hasta -el mayor- con 1,500 hectáreas, suman unas 10.000 hectáreas. En este tramo del río, la auto-gestión informal era típica. Incluso después de la 
transferencia, se mantiene el esquema tradicional de gestión ya que el esquema gubernamental para las nuevas organizaciones (módulos) es demasiado grande y centralizado: hay dos organizaciones formales para los sistemas de riego en este tramo del río. La gestión local, tradicional, no oficial, basada en acuerdos horizontales entre las comunidades ha durado desde los 60's hasta hoy; unos 50 años. (Palerm-Viqueira \& Martínez-Saldaña, 2013).

Tejalpa. El sistema de riego de Tejalpa tiene dos obras de cabecera, aunque los canales estén interconectados. El sistema está caracterizado por una multitud (88) de pequeños depósitos secundarios. El sistema riega unas 1,500 hectáreas, las cuales pertenecen a 29 comunidades y 11 ranchos. La organización formal se puso en marcha en la década de 1930, sin embargo, el marco oficial para la organización desapareció en 1992. Por alguna razón, la política del gobierno sólo está inscribiendo como organización formal a los regantes de cada comunidad. Después de alguna turbulencia, la gestión de todo el sistema sigue intacta. De hecho, la Universidad del Estado ocupa un lugar en el comité (informal) del sistema. (Aguirre, Montes, \& Palerm-Viqueira, 2010).

Nexapa. El comité del río Nexapa abarca 12 sistemas de riego en el tracto del río. Cada sistema tiene una organización formal creada en la década de 1930. El comité se estableció con el fin de regular la distribución del agua, así como para cuidar de la presa y del túnel que transporta agua al río Nexapa desde otro río, el Atoyac. El comité está en vigor desde la década de 1960, es decir, desde hace unos 50 años. Este nuevo nivel organizativo no es oficial y abarca 9,000 hectáreas. En 1992 el marco oficial para los sistemas de riego desapareció, aunque las organizaciones siguen funcionando. Un cambio más reciente es que la organización multi-sistema se ha integrado como Distrito de Riego. Los regantes aceptaron este cambio "en papel", dado que las obras de cabecera para los 12 sistemas (la presa y túnel) necesitaban una reparación considerable, y la Comisión Nacional del Agua sólo tenía presupuesto para los Distritos de Riego. (Rodríguez, 2000; Rendón Pimentel, 2005).

\subsection{La creación de organizaciones, mayormente acuerdos horizontales entre comunidades después de la reforma agraria sin marco legal oficial.}

Valle Coeneo-Huaniqueo. El valle de Coeneo-Huaniqueo utiliza una técnica de riego que se basa en inundar parcelas a su vez rodeadas por diques. El agua puede llegar a tener un metro de profundidad, y se va pasando de una parcela a otra, para finalmente regresar al río. La técnica se denomina entarquinamiento de cajas de agua. Los propietarios de una parcela deciden entre ellos si en un dado año inundan o no el terreno. En una sección del río, hay al menos 2,000 hectáreas de parcelas rodeadas por diques. Los acuerdos horizontales entre las comunidades tienen que ver con descarga del agua para que todas las parcelas puedan beneficiarse del entarquinamiento. No hay ninguna organización formal registrada, aunque los decretos de la reforma agraria indicar mantener los usos y costumbres. La organización horizontal informal ha estado en funcionamiento desde la reforma agraria, hace unos 80 años.

La Cañada. La Cañada, un estrecho valle, cuenta con 19 sistemas de irrigación interconectados. Cada uno de estos sistemas está basado en una presa derivadora y los regantes contribuyen para reconstruirla, un sistema suele desaguar en el siguiente sistema. Es llamativo que un par de los sistemas se expanden durante la temporada de secas, el canal se prolonga haciendo una zanja en el lecho seco del río y se conecta a canales existentes. La gestión se basa en acuerdos horizontales entre las comunidades. En total, el área irrigada abarca unas 
3,000 hectáreas. En la década de 1990, el gobierno creó y registró organizaciones oficiales para alguno de los sistemas; sin embargo, estas organizaciones tienen límites diferentes a los de las organizaciones tradicionales. Los acuerdos informales han estado en vigor desde la reforma agraria, unos 80 años.

\subsection{La creación de organizaciones, mayormente acuerdos horizontales entre comunidades después de la reforma agraria sin marco legal oficial.}

Tepetitlán. El sistema de riego Tepetitlán toma el agua de una presa de almacenamiento. Poco después de la toma, el canal se divide en tres canales principales. El sistema se integró en un Distrito de Riego, probablemente alrededor de la década de 1950; de hecho, la transferencia se vio frenada por que la Comisión Nacional del Agua no pudo encontrar los papeles.

Este sistema riega unas 10,000 hectáreas pertenecientes a 46 comunidades, y fue gestionado por las autoridades del Distrito de riego, aunque las autoridades comunitarias tenían ciertas labores en la distribución del agua a nivel comunidad. En los 90's, con la política de transferencia, los regantes de este sistema rehusaron una costosa organización burocrática. La Comisión Nacional del Agua tuvo que ceder y la transferencia se realizó como unidad de riego (y no como módulo), una solución jurídica lateral para los Distritos de Riego o partes de los Distritos que se negaron a seguir el esquema organizativo propuesto por el gobierno. Lo fascinante de este sistema de riego es el proceso comunitario para tomar el control. El proceso en curso ha sido muy exitoso; por ejemplo, la distribución del agua ha mejorado a pesar de cierta turbulencia interna. El sistema cuenta con un marco legal oficial, pero la gestión no sigue la pauta oficial. En el siguiente cuando se expresan el tamaño de los sistemas y la duración, en años, de organizaciones no-oficiales:

Cuadro 1. Sistemas de riego, superficie y años de informalidad de la organización

\begin{tabular}{|l|c|c|}
\cline { 2 - 3 } \multicolumn{1}{c|}{} & $\begin{array}{c}\text { superficie de regadío } \\
\text { (hectáreas) }\end{array}$ & años de informalidad \\
\hline Juárez & 1,600 & 20 \\
\hline Valle de Santiago & 10,000 & 50 \\
\hline Cuautitlán & 9,000 & 40 \\
\hline Ixmiquilpan & 3,700 & 30 \\
\hline Nazas & 100 a 1,700 & 50 \\
\hline Tejalpa & 1,500 & 20 \\
\hline Nexapa & 8,000 & 50 \\
\hline Coeneo- & 2,000 & 80 \\
Huaniqueo & 3,000 & 80 \\
\hline La Cañada & 9,700 & en curso \\
\hline Tepetitlán & & \\
\hline
\end{tabular}




\section{Análisis}

En la propuesta inicial, supusimos que la continuidad de un marco legal favorable a las organizaciones hacía sinergia y potenciaba las capacidades organizativas; mientras que la discontinuidad del marco legal fragilizaba a las organizaciones. Los datos de los estudios de caso, sin embargo muestran que la organización informal es bastante extensa y exitosa y, por tanto, que existe una falta de coincidencia entre los datos oficiales y las organizaciones reales sobre el terreno. Es decir, el problema puede ser la visibilidad de las organizaciones no oficiales.

\subsection{Aspectos positivos}

Antes de abordar los impactos negativos de los marcos jurídicos cambiantes en la gestión del agua por los mismos regantes, tal vez podríamos abordar algunos aspectos positivos:

3.1.1 La introducción de instituciones unificadas. La introducción de instituciones unificadas permite un foro de negociación y la toma de decisiones consensuadas sobre asuntos que competen a todo el sistema.

A finales del siglo XIX y principios del XX, las políticas públicas y los marcos legales mexicanos introdujeron un diseño organizativo consistente en instituciones unificadas. Anteriormente, la gestión de los sistemas, con la excepción de Valle de Santiago, estaba basada en acuerdos horizontales (notariales o judiciales) entre comunidades y propiedades privadas (haciendas o ranchos), o, en tiempos coloniales, en los cuadros de distribución del agua (repartimientos) realizados por autoridades gubernamentales que intervenían a consecuencia de un conflicto sobre la distribución del agua. Es decir, no había instituciones de regantes que unificaran a las diversas comunidades y propiedades privadas que se beneficiaban del agua del sistema.

3.1.2 Niveles organizativos en las instituciones. La existencia de niveles organizativos en las instituciones es una estrategia de diseño que favorece una gestión no burocrática y empodera a comunidades locales. En la construcción de niveles organizativos la comunidad puede jugar un papel clave.

La reforma agraria y su marco legal re-introdujeron la propiedad común y la gobernanza comunitaria. La comunidad tenía total autoridad sobre la toma de decisiones en su espacio de riego y participaba en la toma de decisiones a nivel del sistema. Este nivel organizativo también es importante para la administración basada en acuerdos horizontales entre comunidades en sistemas de riego multi-comunitarios-tales como los encontrados en varios estudios de caso (Nazas, La Cañada, Coeneo-Huaniqueo).

La capacidad de despliegue organizativa de las comunidades es notable en el caso del sistema de riego Tepetitlán, donde el empuje organizacional después de la transferencia está basado en las comunidades organizadas.

3.1.3 Niveles organizativos sumando organizaciones. La importancia de un diseño basado en niveles organizativos en instituciones unificadas no solo se refiere a empoderar a las comunidades, también se refiere a la capacidad de añadir niveles organizativos y añadir infraestructura y agua a ser administrada. 
En los casos de organizaciones unificadas encontramos que han conseguido añadir un tercer nivel organizacional, cada nivel con labores claramente definidas concernientes a las obras hidráulicas comunes. Estos nuevos niveles, tal como en los casos de Nexapa y Cuautitlán, son informales.

3.1.4 Diseño organizativo basado en niveles organizativos. El diseño organizativo unificado pero basado en niveles organizativos fue una propuesta del gobierno, aunque las más recientes propuestas, desde 1992, eliminan los niveles organizativos. Se puede destacar que la preferencia social parece inclinarse hacia un diseño de varios niveles. Por ejemplo, en la década de 1920, los sistemas del río Cuautitlán rehusaron una gestión centralizada; más recientemente, los regantes del sistema Tepetitlán rechazaron un sistema centralizado (y costoso) de gestión centralizada.

El contraste entre el funcionamiento de diseños organizativos centralizados o con niveles organizativas, aún necesita ser estudiado, especialmente en vista de que, en la transferencia de los Distritos de Riego en la década de los 90, el diseño gubernamental está basado en un modelo burocrático centralizado.

3.1.5 Consideraciones sobre la introducción de instituciones unificadas. La introducción por el gobierno federal de organizaciones unificadas no se ve como positivo por todos los investigadores de México. Hay dos vertientes negativas, la toma de decisiones sobre el agua pasa del nivel estatal al federal a fines del siglo XIX -cambio que se denomina federalización de las aguas, se le da un carácter negativo por trasladar la toma de decisiones sobre agua del nivel local al federal. Es el nivel federal que introduce la obligatoriedad de las organizaciones unificadas, lo que es visto como una "imposición", ya que el gobierno ordena su creación (Aboites, 1998; Valladares, 2004).

Efectivamente el gobierno federal tiene una política, desde finales del siglo XIX pero especialmente en las primeras décadas del siglo XX, de conformación de organizaciones de regantes. La conformación de las organizaciones va de la mano de la obligatoriedad de cuadros de distribución, cuadros que pretenden que todos los usuarios accedan a su derecho de aguas.

La versión negativa de imposición debe matizarse con varios considerandos:

Primero, en diversos países se emite legislación sobre aguas y sobre la conformación de organizaciones de regantes. Segundo, el control sobre el sistema pasa de los municipios o de acuerdos horizontales entre haciendas y comunidades a una sola organización. Es decir el control local se mantiene. Tercero, hay que mirar más allá del momento del mandato de la creación y considerar si hay un proceso de apropiación de la estructura de gobierno por los mismos regantes. Es decir, cuestiones como si los regantes piensan que la organización es de ellos, si responde a sus necesidades, cuántos retoques se han hecho a la estructura de gobierno original propuesta por el gobierno, y hasta qué punto cumple con los principios de diseño de Ostrom.

\subsection{Aspectos negativos}

Los siguientes puntos son sobre los aspectos negativos de los cambios en las políticas públicas y los marcos legales. 
3.2.1 Expansión de la gestión directa por el Estado federal. El primero en la lista es la expansión de la gestión directa del gobierno. La conveniencia o la necesidad de gestión gubernamental en los nuevos proyectos de riego no están bajo discusión, más bien aquellos casos en los que ya había una gestión de regantes en existencia, y que fue desplazada por la gestión del gobierno. Es negativo, cuando menos, en que desplaza el auto-gobierno.

3.2.2 Capacidad de resistencia a la expansión de la gestión directa por el Estado federal. En el caso de México la resistencia de los regantes a la ampliación de la gestión por el Estado está mal documentada. En algunos casos, tales como el de las organizaciones de Ixmiquilpan o Juárez, la resistencia abarcó todo el sistema; en otros, tales como el de los sistemas de irrigación del río Cuautla, la resistencia sólo fue efectiva en la distribución del agua a nivel comunidad (Ávalos, Aguilar Sánchez, \& Palerm-Viqueira, 2010). En el Distrito de Riego de la Comarca Lagunera, la autogestión de los pequeños sistemas de riego en la parte alta del río Nazas parece haber persistido debido a la negligencia benigna que permite el Distrito de Riego, ya que eran marginales y distantes de la superficie principal de riego.

No parece existir una salida judicial, es decir donde los regantes pudieran oponerse en los tribunales a la apropiación de la gestión por el Estado. Es un espacio que no parecer otorgar la legislación.

La documentación de la resistencia de los regantes ante la expansión de la gestión gubernamental es un tema interesante para su seguimiento. Arthur Maass (Maass \& Anderson, 1976) argumentaron que la capacidad de los regantes de auto-gobernarse y negociar con el Estado demostraba los límites de la hipótesis hidráulica de Wittfogel (Wittfogel, 1957). Desde otra perspectiva, Sengupta (Sengupta, 1993) ha argumentado que la apropiación por el Estado de la gestión es más fácil en grandes que en pequeños sistemas aislados, por la facilidad de implantar una administración burocrática costeable.

3.2.3 Cambios en la nomenclatura de las organizaciones de regantes. Hay cambios en la nomenclatura de las organizaciones de regantes: asociaciones de usuarios, asociaciones de regantes, juntas de aguas, urderales (unidades de riego para el desarrollo rural), unidades de riego, módulos.

Para algunas organizaciones, la actualización aparentemente significo sólo un cambio de nombre (junta de aguas, urderal, unidad de riego) -y no todas las organizaciones pasaron por todos los cambios de nombre.

En el cambio de nombre, varias organizaciones de regantes aceptaron solo bajo la condición de que nada cambiaría (como por ejemplo en los casos de Ixmiquilpan y Nexapa), y otras organizaciones aún se refieren a sí mismas por el nombre anterior, usualmente junta de aguas (por ejemplo, en el caso de Cuautitlán).

Sin embargo, en algunos casos, la falta de actualización significó que la organización pasó de ser formal a informal. En otros casos, aunque hubo una actualización de la organización, los límites de una organización de regantes dada cambiaron; como por ejemplo en el caso de Tejalpa, en donde nuevas organizaciones fueron establecidas a nivel comunidad (reemplazando la gestión por las autoridades del ejido por una gestión por un comité nombrado únicamente por los regantes), pero no hubo ninguna sustitución de la organización multicomunitaria del sistema. La impresión general es que, para la gestión de todo el sistema, el cambio de nombre ha sido irrelevante para los regantes. 
3.2.4 Desplazamiento del ejido e individualización derechos de agua. El desplazamiento del ejido como autoridad sobre el agua va de la mano de la individualización de los derechos de agua. Estos cambios pueden tener consecuencias a largo plazo, en los Distritos de Riego la renta y venta a muerte de derechos de agua por parte de los ejidatarios es notorio (Contreras-Rentería, 2001; Fortis-Hernández \& Alhers, 1999).

3.2.5 Fragilización. Mientras buscábamos indicadores de fragilización en la organización de los sistemas de mayor tamaño, hubo una creciente impresión de la posibilidad de que las organizaciones podrían colapsarse desde abajo. La organización a nivel comunitario parece ser un elemento fundamental para las grandes organizaciones, como un nivel organizativo en las organizaciones unificadas. También es un elemento fundamental en redes organizativas, tales como los acuerdos horizontales entre comunidades. El empuje para tomar control de la gestión y para construir una organización parece residir en la organización a nivel comunidad, el caso de Tepetitlán es ejemplar en este sentido.

3.2.6 Resistencia. Las comunidades pueden, por supuesto, poner resistencia a individualización y al desplazamiento de la toma de decisiones comunitarias. El reciente marco legal que centraliza el agua de uso doméstico en ámbito municipal ha llevado a las comunidades a resistir la centralización (Galindo-Escamilla \& Palerm-Viqueira, 2007; López-Villamar, Martínez Saldaña, \& Palerm-Viqueira, 2013). Sin embargo, estas situaciones llevan a una posición defensiva frente a los marcos jurídicos nacionales y las políticas públicas, en lugar de a la sinergia.

\subsection{Más investigación}

Los marcos legales de México y las políticas públicas se están moviendo en dirección hacia la descentralización, la transferencia de los Distritos de Riego, y la (supuesta) descentralización de los sistemas de abastecimiento para uso doméstico.

No obstante,

1) la organización propuesta para la transferencia de los Distritos de Riego está basada en una organización burocrática centralizada, con escasa participación de las comunidades.

2) la centralización en el municipio de la administración de sistemas de agua potable comunitarios, significa un desplazamiento de la participación comunitaria y usualmente una burocratización de la gerencia.

¿Cómo funcionan estos diseños organizativos?, ¿son más eficaces? ¿Hay economías de escala? ¿Hay una profesionalización con impactos positivos?

\section{Referencias}

Aboites, L. (1998): El agua de la nación. Una historia politica de México (1888-1946). CIESAS.

Aguirre, N., Montes, R., \& Palerm-Viqueira, J. (2010): Historia del manejo social del agua en la subcuenca del río Tejalpa, Estado de México. In Memorias I Congreso Red-ISSA, IMTA, 
18 y 19 de marzo de 2010. Presented at the I Congreso de la Red-ISSA, Jiutepec: IMTA. Retrieved from http://redissa.hostei.com/rissa/Mesas_2010.htm

Arteaga, L. (1931): Algo más sobre las agrupaciones de regantes. Irrigación en México, 3(4), 312-319.

Ávalos, C., Aguilar Sánchez, G., \& Palerm-Viqueira, J. (2010): Gestión técnica y social del uso del agua en Morelos: caso del río Cuautla. UACh.

Contreras-Rentería, R. M. (2001): El costo social del rentismo de derechos de agua superficial y tierras de riego en la región lagunera (Tesis Maestría). Colegio de Postgraduados.

Erickson, C. L. (2006): Intensification, Political Economy, and the Farming Community; In Defense Of A Bottom-Up Perspective Of The Past. In J. Marcus \& C. Stanish (Eds.), Agricultural Strategies (pp. 334-363). Los Angeles: Cotsen Institute.

Fortis-Hernández, M., \& Alhers, R. (1999): Naturaleza y extensión dle mercado del aguaen el D.R. 017 de la Comarca Lagunera, México. México: Instituto Internacional del Manejo del Agua.

Galindo-Escamilla, E., \& Palerm-Viqueira, J. (2007): Pequeños sistemas de agua potable, entre la autogestión y el manejo municipal en el estado de Hidalgo, México. Agricultura. Sociedad y Desarrollo, 4(2), 127-146.

Garma, F. (2006): Breve reseña de los derechos de Laborío del valle de Santiago, Gto. a las aguas del río Lerma y laguna de Yuriria (1921). In I. Santos (Ed.), Riego y gestión del agua en la cuenca Lerma-Chapala. Documentos para su Historia. 1896-1985 (DVD). SEMARNAT, IMTA, AHA.

Glick, T. (1970): Irrigation and society in Medieval Valencia. Cambridge, Mass.: Harvard University Press.

Hunt, R. (1997): Sistemas de riego por canales: tamaño del sistema y estructura de la autoridad. In J. Palerm-Viqueira (Ed.), Antología sobre pequeño riego (Vol. 1, pp. 185-219). México: Colegio de Postgraduados.

Hunt, R. (2007): Communal irrigation, a comparative perspective. In $A$ World of Water Rain, rivers and seas in Southeast Asian bistories (pp. 187-208). Leiden: KITLV Press (Koninklijk Instituut voor Taal-, Land- en Volkenkunde). Retrieved from http:/ /www.oapen.org/

Hunt, R., Guillet, D., Abbott, D. R., Fish, P., Fish, S., Kintigh, K., \& Neely, J. A. (2005): Plausible ethnographic analogies for the social organization of Hohokam canal irrigation. American Antiquity, 70(3), 433-456.

López-Villamar, S., Martínez Saldaña, T., \& Palerm-Viqueira, J. (2013): Las comunidades en la administración de sistemas de agua potable: región de los volcanes, estado de México. Agricultura, Sociedad y Desarrollo, 10(1), 39-58.

Maass, A., \& Anderson, R. L. (1976): ... and the desert shall rejoice. Conflict, growth and justice in arid environments. Cambridge: The MIT Press.

Ostrom, E. (1993): Design Principles in Long-Enduring Irrigation Institutions. Water Resources Research, 29(7), 1907-1912.

Ostrom, E. (2009): Design Principles of Robust Property Rights Institutions: What Have We Learned? In G. K. Ingram \& Y.-H. Hong (Eds.), Property Rights and Land Policies (pp. 25-51). Cambridge, MA: Lincoln Institute of Land Policy.

Ostrom, E. (2011): El gobierno de los bienes comunes. La evolución de las instituciones de acción colectiva. México: Fondo de Cultura Económica/SEMARNAP. 
Palerm-Viqueira, J. (2000): Administración de sistemas de riego: tipos de autogestión. In $X$ Memorias X Congreso Nacional de Irrigación. Chihuahua: Asociación Nacional de Especialistas en Irrigación.

Palerm-Viqueira, J. (2001): Organizational strategies in water shortage situations: Mexican self-administrated irrigation systems. International Journal of Water, 1(3-4), 285-306.

Palerm-Viqueira, J. (2005): Políticas del Estado para la administración y gobierno de sistemas de riego y redes hidráulicas. In J. M. Durán, M. Sánchez, \& A. Escobar (Eds.), El agua en la historia. Balance y perspectivas. (pp. 263-289). AHA/ Colegio de Michoacán/ Universidad de Guadalajara/ CIESAS.

Palerm-Viqueira, J. (2006): Self-Management of Irrigation Systems, a Typology: The Mexican Case. Mexican Studies/Estudios Mexicanos, 22(2), 361-385.

Palerm-Viqueira, J. (2009): Distritos de riego en México, algunos mitos. In J. PalermViqueira \& T. Martínez Saldaña (Eds.), Aventuras con el agua. La administración del agua de riego: historia y teoría (pp. 277-328). México: Colegio de Postgraduados.

Palerm-Viqueira, J. (2010): Comparative history of irrigation water management, from the sixteenth to twentieth centuries: Spain, Mexico, Chile, Mendoza (Argentina) and Peru. $W a$ ter Policy, 12, 779-797.

Palerm-Viqueira, J., y Martínez-Saldaña, T. (Eds.). (2013): Antología sobre riego: Instituciones para la gestión del agua: vernáculas, alegales e informales (en prensa.). Biblioteca básica de agricultura, Colegio de Postgraduados/ Grupo Mundi-Prensa.

Palerm-Viqueira, J., Sandré, I., Rodríguez-Haros, B., \& Caletteet, N. D. (Eds.). (2004): Catálogo de Reglamentos de Agua en México. Siglo XX. AHA/ CIESAS/ CNA.

Rendón Pimentel, L. (Gerente N. de D. de R. (2005): Nexapa, Distrito de Riego.

Rodríguez, G. (2000): El Comité de Vigilancia de los ríos Atoyac y Nexapa: formación de una organización. In J. Palerm-Viqueira \& T. Martínez Saldaña (Eds.), Antología sobre pequeno riego. Organizaciones autogestivas (Vol. 2, pp. 345-406). Colegio de Postgraduados/ Plaza y Valdés.

Rodríguez-Haros, B., \& Palerm-Viqueira, J. (2007): Before transference: handing over Water Districts/ Antes de la transferencia: la entrega de Distritos de Riego. Agricultura. Sociedad y Desarrollo, 4(2), 105- 126.

Sandré, I. (2012): Entre la ley y la costumbre. tradición y poder local en la gestión del sistema de riego del Río Cuautitlan, Estado de México (Doctorado Antropología). UAM-Ixtapalapa, Mexico.

Sandré, I., \& Sánchez, M. (Eds.). (2011): El eslabón perdido. Acuerdos, convenios, reglamentos y leyes locales de agua en México. CIESAS.

Santos, I. (Ed.) (2006): Riego y gestión del agua en la cuenca Lerma-Chapala. Documentos para su Historia. 1896-1985 (DVD). SEMARNAT, IMTA, AHA.

Sengupta, N. (1993): User-friendly Irrigation Designs. New Delhi, India: Sage Publishers.

Sengupta, N. (2002): Institutions and Engineering. Evolution of Irrigation Designs In Some Asian Countries. In Conference on the Environmental History of Asia. Presented at the Conference on the Environmental History of Asia, University of Sussex.

Vaidyanathan, A. (2009): Instituciones de control del agua y agricultura: una perspectiva comparativa. In J. Palerm-Viqueira \& T. Martínez Saldaña (Eds.), Aventuras con el agua. La administración del agua de riego: historia y teoría (pp. 73-115). 
Valladares, L. (2004): Conflictos hidráulicos en Morelos 1880-1940: de la hacienda al modelo ejidal campesino. Boletín Archivo Histórico del Agua, número especial Organizaciones autogestivas para el riego, nueva época año 9, publicación de aniversario (10 años), 67-79.

Wittfogel, K. (1957): Oriental despotism. A comparative study of total power. New Haven and London: Yale University Press. Retrieved from http://ia600702.us.archive.org /2/items/KarlAugustWittfogel-OrientalDespotism/KarlAugustWittfogel-OrientalDespotism.pdf

Worster, D. (1985): Rivers of Empire. Water, Aridity and the growth of the American West. New York: Pantheon Books. 